\title{
Publicidad y consumo simbólico
}

\section{Juan A. González*}

\author{
Universidad Complutense
}

\section{PLANTEAMIENTO}

Apenas nadie duda ya de la irracionalidad aparente de nuestras conductas de consumo, ahora bien, esta irracionalidad conforma racionalidades colectivas, que deben estudiarse y describirse. Los objetos de consumo, como síntesis de la subjetividad de productores y consumidores, son el instrumento más seguro para descubrir estas racionalidades. Consumir ha dejado de ser una actividad sencilla, determinada por las necesidades de los sujetos, por sus respectivos niveles de renta y por la utilidad concreta de la transacción, para transformarse en un complejo proceso de interactuación y reproducción social, donde confluyen múltiples variables que dificultan su comprensión y predictibilidad. La mayoría de objetos se subjetivizan en el consumo, valiendo más por lo que representan, es decir, por su capacidad semiótica, que por lo que realmente son, esto es, por su funcionalidad: además se orientan a la satisfacción de necesidades no siempre bien definidas, generadoras de consumo simbólico. Por su parte, los niveles de renta se amplían y generalizan como consecuencia lógica de la sociedad de servicio, que nos presiona con su permanente oferta en favor de un consumo fácil, personalizado y repetido, y en detrimento, no sólo del ahorro, sino incluso de nuestra renta potencial futura y por tanto de una planificación económica, social y política más eficaz ${ }^{1}$. Por último, la utilidad que se busca en el consumo es polivalente y se acomoda no sólo a las decisiones y posibilidades personales, sino también al potencial comunicativo de los objetos, a la información que recibe el consumidor, a las conductas grupales y sociales, a la influencia del contexto... siendo muy variadas las causas que originan una conducta de consumo.

Ante esta compleja situación no sirven modelos explicativos parciales e inadecuados por su simpleza o por su ambigüedad. El consumo es un proceso de naturaleza sincrética, que precisa un enfoque multidisciplinar e integrado, capaz de valorar el modo como las distintas explicaciones se complementan. Seis son las perspectivas de análisis que han tratado de explicarlo: las teorías económicas, cuyo origen se remonta al s. XVIII, los modelos conductistas, la «motivational research», y el paradigma cognitivo, que coinciden en la orientación psicológica, aunque la naturaleza de sus explicaciones son muy diversas; el enfoque sociológico, bastante más difícil de adscribir a escuelas o tendencias concretas; y la aproximación semiótica, sobre la que se centrará el grueso de mi aportación. No es objeto de este artículo hacer una descripción exhaustiva de cada uno de estos planteamientos, sino más bien tratar de desvelar el modo como se produce este proceso; es conveniente, en cualquier caso, indicar algunas de sus limitaciones, para justificar el alcance de esta propuesta.

* Dirección del autor: Universidad Complutense, Facultad de Ciencias de la Información, Avda. Complutense s/n. 28040 Madrid. 
Los planteamientos economicistas (G. Katona 1960, 1975; F. M. Nicosia 1966, 1968; H. A. J. Green, 1971) se basan en la racionalidad del bomo ceconomicus, entendida como una síntesis abstracta de las decisiones individuales dominantes, y en la coherencia reflexiva de su comportamiento orientado al beneficio y al empleo adecuado de la renta. No explican, a pesar de la complicación de algunos de sus modelos, más que parcialmente y por tanto de modo deformante este proceso: la irracionalidad (H.A.J. Green 1971) crea tendencias racionales que condicionan el mercado, aunque frecuentemente se busca justificación racional para conductas que hace tiempo dejaron de serlo, como por ejemplo todo el consumo alimentario. Esta aproximación al consumo se invalida no sólo por su parcialidad, sino principalmente porque se ve obligada a acudir a otros modelos para entender su propio funcionamiento (J. K. Galbraith, 1958, 1967, 1977; J. P. Reynaud 1974; K. E. Warneryd 1980 y G. M. van Veldhoved, 1980).

Tampoco modelos reductores y rígidos como los conductistas (J. F. Engel, D. T. Kollat y R. D. Blackwell 1968; J. A. Howard y J. N. Shert 1969; A.S.C. Ehrenberg 1972 o D. A. Aaker y S. M. Morgan 1971), que conciben al consumidor como un sujeto pasivo y condicionado, que asimila los hábitos de consumo de manera refleja, son satisfactorios por cuanto se refieren a un consumidor arquetipo, fruto del funcionamiento cuantitativo del mercado y de la extrapolación de conductas simples a situaciones más complejas de interactuación, olvidando que no somos elementos neutros de este proceso, sino que vivimos inmersos en una verdadera red de comunicaciones, de mensajes coincidentes o complementarios, en donde se desarrolla nuestra vida individual, familiar, social y política. Los automatismos y rutinas del consumo, verdadero caballo de batalla de esta escuela, si es que existen, son más frágiles y complejos de lo que afirman los conductistas y se mezclan con implicaciones emotivas, afectivas, valores..., impidiendo el aprendizaje mecánico de cualquier conducta de consumo.

Los planteamientos psicoanalíticos, una de cuyas aportaciones es la llamada «motivational research» ${ }^{2}$, tal como se entiende en E. Dichter 1961, J. Marcus-Steiff 1961, 1963, P. Martineau 1969 o D. Wulf 1975, se orientan a descubrir la irracionalidad de gran parte de la conducta de los consumidores, dirigida a aplacar ilusoriamente la angustia y llenar el vacío existencial del individuo (E. Fromm 1960); el consumo genera comportamientos perversos, en los que no se corresponde el instinto desencadenante de la conducta con el objeto de ésta, y psicopatologías (F. Dogana 1980) causadas por las sugestiones y representaciones del propio consumidor, quien simboliza a los objetos conforme a sus fantasías. Es una concepción imaginaria del consumo que no debe confundirse con el consumo simbólico, pues se usa para interpretar los modos como el individuo representa la sociedad a través de las desviaciones de su conducta de consumidor y para corregir posibles patologías. De todos modos, esta aproximación responde inadecuadamente a la amplia problemática psicológica originada en torno a este proceso. Además, sus instrumentos (reuniones de grupo sin directrices, entrevistas en profundidad, técnicas proyectivas...) se adaptan mejor a la virtualidad de algunos de sus cultivadores, que a la accesibilidad de los métodos científicos.

Para tratar de superar las limitaciones descubiertas en las dos aportaciones anteriores surge el paradigma cognitivo ${ }^{3}$ que se ocupó inicialmente de la formación y cambio de las actitudes como punto de arranque de la conducta del consumidor. El conocido modelo expectativa-valor de $\mathbf{M}$. J. Rosenberg y M. Fishbein (R. J. Lutz y J. R. Bettman 1977 y F. Carrera 
1979) supuso una aportación a la explicación de esta conducta , aunque no pudo zafarse del carácter reductivo que le imponía su herencia conductista, olvidando variables tan importantes como la influencia grupal o las incidencias contextuales. El modelo ampliado actitud-conducta (I. Ajzent y $\mathbf{M}$. Fishbein 1981) pretende subsanar estas deficiencias valorando la actitud del consumidor en relación con la eficacia de ciertas conductas, buscando combinar influencias cognitivas individuales con las interpersonales y grupales ${ }^{4}$; este modelo ha interesado poco por su naturaleza genérica y por su baja capacidad predictiva. La investigación cognitiva, olvidando esta consecuencia neoconductista, se ocupa ahora de las respuestas cognitivas concretas y de los procesos persuasivos que las generan: la fuente de la información, el modo como se presentan los mensajes y las condiciones de recepción son las variables que determinan la conducta del consumidor ( $\mathrm{J}$. N. Kapfferer 1978); preocupa a los psicólogos cognitivos el modo en que el consumidor procesa la información que recibe, llegándose a conclusiones tan desconcertantes para los defensores de la educación para el consumo, como las de J. Jacoby, W. D. Hayer y D. A. Sheluga 1981, que muestran cómo demasiada información conlleva una peor elección de compra y consumo. Conviene aclarar que la publicidad es la principal fuente de información para una mayoría de consumidores y se caracteriza por ser un sistema muy poco informativo; por lo que hay que acudir a otros enfoques, que aclaren el modo en que el consumidor recibe y transmite sentidos dejándose influir no tanto por el contenido denotado de los mensajes, como por el efecto connotado de objetos, grupos y contextos. Aún así el paradigma cognitivo of rece otras posibilidades explicativas, como muestra el trabajo de P. Vidal, integrado en esta monografía.

Los enfoque sociológicos coinciden en el análisis de un consumidor conformado, integrante de varios grupos sociales que imponen sobre él valores y normas. El individuo se comporta en relación con su identidad social, orientando su conducta de acuerdo con la conducta social de los grupos a los que pertenece.

El marco social - dice $\mathrm{H}$. Tajfel- de las relaciones intergrupales contribuye a hacer a los individuos lo que son; éstos, a su vez, producen ese marco; ambos se desarrollan y cambian simbióticamente.

(H. Tajfel 1984, pág. 52).

La imitación, la expresión de estatus, los valores y los estilos de vida (B. Chatelat 1980) son el origen de la conducta social del consumidor; el consumo se reviste de las propiedades de un grupo: cada grupo social produce sus propios signos, que se decodifican como significantes, es decir, como representantes, de este grupo, identificado socialmente por este universo semiótico. La sociedad produce y se reproduce a través de este universo de signos y aquí coinciden el consumo integrativo y el simbólicos, diferenciándose en que el primero es el consumo de la abundancia, de la producción masiva y del derroche y una vez que ha cambiado profundamente el contexto general por la crisis energética y de materias primas, por el desarrollo tecnológico y por el descontrol social, referentes y grupos se enmascaran y se necesita un nuevo enfoque para el análisis del consumo. Los productos no son más que significantes vacíos que se llenan de significados culturales de cara al consumo, integrándose objetos y sujetos en el acto de consumir: todo símbolo implica un proceso cognitivo completo donde a través de signos concretos, en este caso los productos, se pasa a un universo cultural socialmente asumido por los sujetos desde la infancia; el símbolo es pluridimensional de modo que evoca subjetivamen- 
te los componentes múltiples, reales o atribuidos, de un objeto; de estas evidencias arranca la perspectiva sincrética que ahora propongo ${ }^{6}$. Este planteamiento parte, por tanto, del mundo materializado de la producción para llegar al universo simbolizado del consumo, pretendiendo describir el funcionamiento de todo el sistema, no sólo en tanto fabrica y consume objetos, sino principalmente en cuanto que trata de producir y consumir a los propios consumidores.

\section{VARIABLES QUE DEFINEN LA NOCION DE CONSUMO}

Gracias a la superproducción que sufre nuestra sociedad desde mediados de siglo, el consumo se ha vuelto un indicador inestimable para descubrir no sólo el nivel de desarrollo, sino también nuestro comportamiento social. Los intereses políticos, económicos y sociales más diversos coinciden en defender al consumo como la única posibilidad de que nuestro mundo pueda seguir reproduciéndose, bajando incluso los impuestos para aumentar la capacidad consumidora. No es momento de cuestionarnos esta propuesta, los periódicos día a día reflejan algunos de sus efectos: desastres ecológicos, superexplotación del Tercer Mundo, colonialismo, derroche energético...; pero para abordar la comprensión de este concepto parece inevitable definir los componentes básicos de esta ideología: toda acción de consumo es, básicamente, un acto de elección, es decir, una manera de responder a los mensajes que sustituyen a los objetos, y por tanto, una forma de comunicación y de cultura.

Por eso, resultan inaceptables concepciones simplificadoras como la keynesiana ${ }^{7}$ que equipara el consumir con la obtención de utilidad de un bien, adquirido por el consumidor con la idea de satisfacer ciertas necesidades; a medida que estas se generan psico-socialmente disminuye la posibilidad de conocer la utilidad de los productos y la saciabilidad de los deseos, aumentando la capacidad de consumo (J. K. Galbraith 1968, pág. 188 y ss.) que se abre hacia el infinito, precisando otros enfoques para su comprensión y análisis, de modo que no se generalice, confunda o malinterprete este concepto.

No se puede olvidar tampoco que gran parte del consumo se desarrolla en una civilización del ocio, lo que explica la proliferación desmesurada de servicios y el sentido lúdico con el que se reviste todo el proceso, donde el tiempo se entiende como un valor de cambio, que puede condicionar las conductas de los sujetos, transformándose en una variable importante para las clases más acomodadas (A. Miguel 1983), aunque para una inmensa mayoría su sentido último no es otro que la libertad de perderlo.

La utilidad del consumo se mide hoy en relación con el grado de deseabilidad social y cultural de los objetos integrados en un : intercambio simbólico, cuyos efectos son difícilmente valorables. El comportamiento de los consumidores llena de incertidumbres el mundo de los negocios, pues la demanda de los objetos no depende ya de un sistema de competencia perfecta, sino de un conjunto de variables exógenas que la investigación mercadotécnica y publicitaria ayuda a controlar.

El consumo es, por tanto, la consecuencia de la conjugación de tres tipos de variables:

- Unas objetivas, determinadas por los recursos y limitaciones que impone el entorno y por el grado de funcionalidad de los objetos.

- Otras subjetivas, dependientes de la personalidad individual y social de cada consumidor y del modo como interactúa con su contexto. 
- Finalmente, existe un tercer grupo, de naturaleza tanto objetiva como subjetiva, que se corresponde con el sistema de los objetos y se orienta hacia la representación y la significación.

Este proceso general puede esquematizarse así:

CuADRo I

El consumo y sus variables
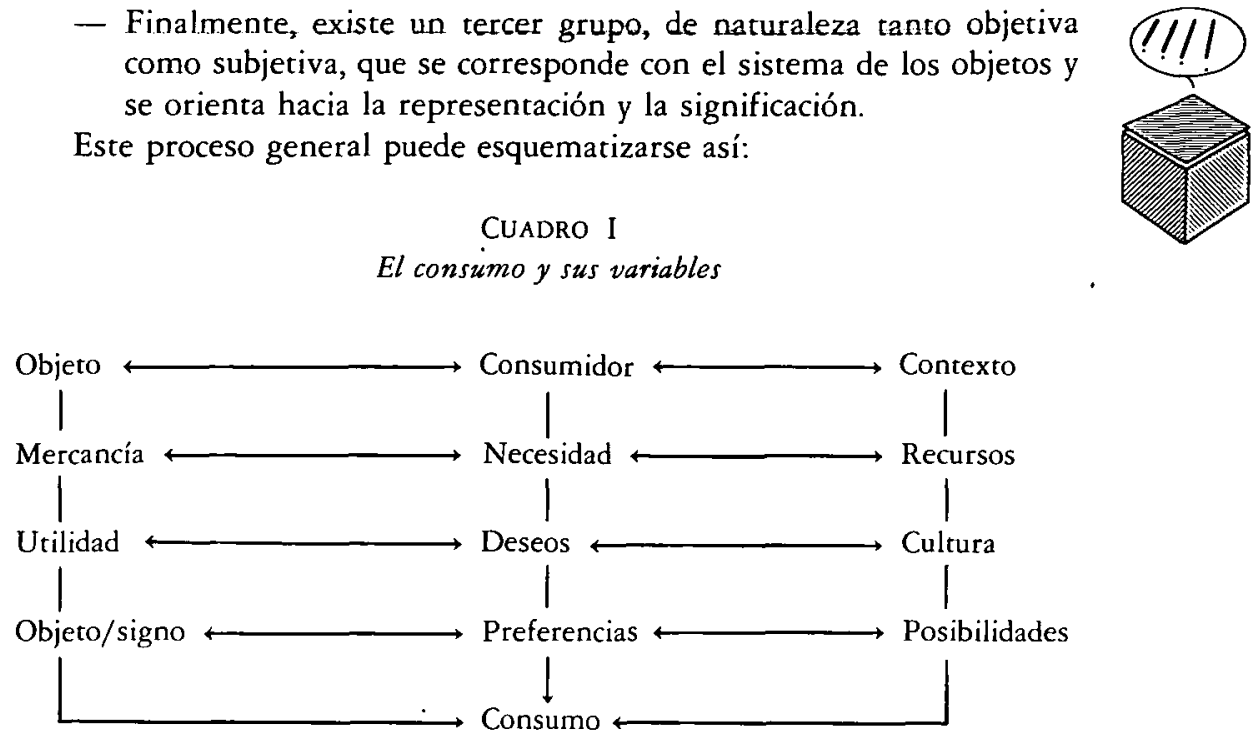

Las decisiones de los consumidores, por tanto, se relacionan con tres aspectos: con las limitaciones originadas por las disponibilidades de recursos cada vez más escasos ante una enorme variedad de medios alternativos; con el modo como éste organice su universo cognitivo, origen frecuente de la demanda no funcional y del consumo ostentoso ( $T$. Veblen, 1944); con el contexto comunicativo que rodea a los objetos. El consumo se encuentra delimitado por el mundo de los objetos, significantes vacíos de un enorme proceso de comunicación; por el universo de los deseos, gustos y preferencias de los consumidores y por las restricciones contextuales, que deberían llenar de racionalidad a todo el sistema. El consumo es efecto y causa del sistema productivo, pero también un acto de elección donde el consumidor se ilusiona y se integra socialmente: el consumo es el instrumento que tiene la sociedad para reproducirse simbólicamente hasta el infinito.

\section{HACIA UNA COMPRENSION INTEGRADA DEL CONSUMO COMO ACTO DE COMUNICACION: EL CONSUMO SIMBOLICO}

Como terminamos de ver, el consumo no puede entenderse solamente como una satisfacción pasiva de necesidades, ni como un comportamiento automático conforme a los dictámenes del mercado, ni como una interpretación imaginaria de la realidad, ni como una interiorización de conductas cognitivas, ni como una conformación social.

El consumo - como indica acertadamente J Baudrillard - no es ese modo pasivo de absorción y de apropiación que oponemos al modo activo de la producción para poner en equilibrio esquemas ingenuos de comportamiento (...). El consumo es un modo activo de relación (no sólo con los objetos, sino con la colectividad y el mundo), un modo de actividad sistemática y de respuesta global en el que se funda todo nuestro sistema de cultura. (J. Baudrillard 1968, pág. 223). 
Para comprender este enfoque, en el que importa menos la capacidad material de consumir, que la predisposición y la deseabilidad de interactuar simbólicamente, hay que distinguir cuatro clases diferentes de lógicas en la producción y reproducción modernas:

- La lógica funcional del valor de uso, de naturaleza operacional y práctica, que busca exclusivamente la utilidad de los productos.

- La lógica económica del valor de cambio, que se preocupa ya por responder adecuadamente a los requerimientos del mercado, a través del control de la relación de equivalencia de los productos. De estas dos lógicas se han ocupado los modelos económicos.

- La lógica del cambio simbólico, que trata de añadir un valor representacional al objeto, vaciándolo de materialidad y llenándolo de contenidos culturales.

- La lógica del valor/signo, a través de la que se transforma el objeto en vehículo de mensajes diversos, que le diferencian artificialmente de otras producciones y lo integan dentro del sistema general de reproducción.

El consumo es una consecuencia de la combinación de estas cuatro lógicas (J. Baudrillard 1972). Los objetos se someten a un proceso de desmaterialización, donde llegan a perder sus características más propias: el café la cafeína, los caramelos el azúcar, la cerveza el alcohol... para adaptarse mejor a las subjetividades de los consumidores. Se personalizan a través de la marca y de la imagen que alrededor de esta se crea, dejando de ser cosas para transformarse en representaciones, de este modo consumimos caricias, frescura, aceptación social en vez de productos de cosmética, naturalidad, dietética y salud en vez de alimentos, confort, liberación y tiempo libre en vez de electrodomésticos... Todo ello de acuerdo con el esquema funcional del lenguaje publicitario (J.A. Gónzález 1982), que puede representarse así:

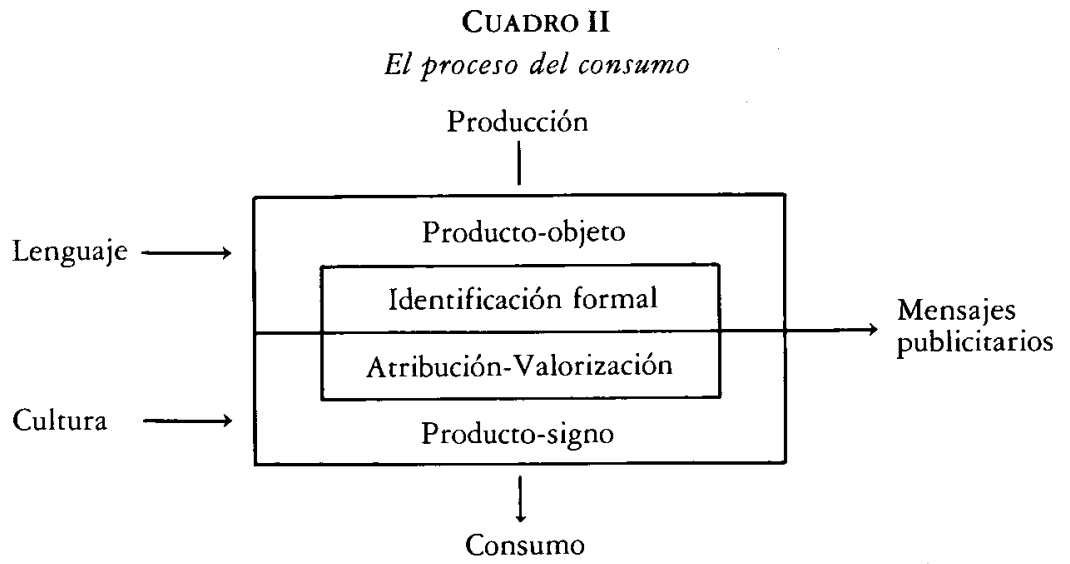

Por medio de las marcas se estratifica y jerarquiza el consumo, iniciándose la valorización de los productos; la marca supone el paso de la objetividad del producto al simbolismo de su personificación; marcando no sólo a los objetos, sino a los propios consumidores (J. Ibáñez 1979), que se identifican con determinadas imágenes de marca, esto es con ciertas configuraciones de cualidades, reales o imaginarias, de los productos. La atribución construye un sentido, una personalidad, una valoración positiva, 
que se asocia a la marca. De este modo el consumo no se agota en los objetos, sino que se interioriza en el universo cognitivo de los consumidores, que intentan, a través del uso de determinados paquetes atributivos, integrarse o imitar a los grupos consumidores de ciertas marcas.

Efectivamente, el consumo se promueve gracias a este cambio y funciona como una sistemática manipulación de signos. En toda acción de consumo destacan dos procesos fundamentales:

- Uno de comunicación y significación, fundado en el uso de unos códigos cada vez más sofisticados (publicidad, diseño, «merchandising», «packansing»...) que pretenden llenar de sentido a las transacciones económicas.

- Otro de jerarquización y de diferenciación social, en el que los productos (objetos-signos) funcionan no sólo como signos, sino como valores dentro de una determinada escala social.

El consumo por tanto se expresa a través de un universo simbólico y expresa una determinada estratificación social, aunque en España lo masivo suele revestirse de aristocrático y la moda la imponen los grandes almacenes y la «fast food» se acepta como la única alimentación juvenil, haciendo de la horterada exhibición ostentosa (A. Miguel 1983), lo que enriquece más aún el intercambio simbólico. Los objetos se adquieren para usarse, pero principalmente para exhibirse como indicadores de ciertas identidades sociales o como modo de opulencia. En cualquier caso, el proceso se origina menos para satisfacer necesidades (consumo funcional) que para soportar significaciones (consumo no funcional). La necesidad se ha vuelto deseabilidad social y el consumo simbólico funciona como un sistema de comunicaciones altamente reproductivo; las mercancías son el gran medio de comunicación de la posmodernidad. «Pero el mensaje que los objetos transmiten - aclara J. Baudrillard- por su mediación ya está simplificado en extremo y es siempre el mismo: su valor de intercambio. Así, en el fondo el mensaje ya no existe; es el medio el que se impone en su pura circulación (J. Baudrillard 1985, pág. 194).

Algunas de las consecuencias de este consumo, que caracteriza a nuestra sociedad posmoderna, son:

- La personalización de los objetos y de los servicios. El principio y fin de toda actividad económica es el $t u ́$ individual; se mercantilizan todos los modos de vida, de forma especial los gustos, deseos y preferencias del posible consumidor, que es asediado y seducido por el hedonismo del consumo ${ }^{8}$.

- La propuesta universal del goce inmediato, que diferencia nuestra sociedad de la industrial, más planificada y racional, y que enmascara a los intereses que subyacen bajo el consumo de la abundancia; una sociedad muy productiva precisa un consumo derrochador y omnipresente, que reproduce necesidades, deseos, valores... como si se tratase de simples mercancías.

- De este modo, toda la sociedad está orientada hacia el consumo, siendo los objetos y su universo simbólico los que generan la sociedad y no al contrario. En la vieja economía industrial se creaban exclusivamente mercancías, la economía del consumo crea principalmente consumidores y asombra ver cómo los fabricantes pueden llegar a gastar más dinero en la promoción de sus productos, que en su producción.

- El consumo presenta una ideología de apariencia democrática, basada en una dinámica social donde se asciende por la capacidad de consumo y en la que todo el mundo puede participar. De este modo

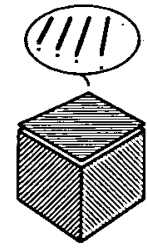


no se distingue la ideología del consumo del acto mismo de consumir y la sociedad se reproduce fabricando en serie modelos de comportamiento social.

- Utilización de los sujetos como instrumentos de consumo y no como fuerza productiva. El consumo origina, según mi opinión, un mecanismo de poder contra el que debemos situarnos, ya que en esto se apoya nuestra colaboración con el sistema económico, social, político y cultural imperante.

En suma, pues, el consumo objetualiza a las personas y personaliza a los objetos. La sociedad industrial fabricaba y consumía productos, nuestra sociedad post-industrial produce y consume consumidores.

El capitalismo de producción - puntualiza J. Ibáñez - tomaba como datos las necesidades: producía los productos que satisficieran esas necesidades e informaba a los consumidores sobre esos productos. El capitalismo de consumo toma como datos los productos: produce las necesidades de consumir esos productos y forma a los consumidores para ello (J. Ibáñez 1985, pág. 5).

En el consumo simbólico el acto de consumo es más importante que el consumidor y el producto pierde terreno frente a los mensajes que lo decoran; por ello me ha parecido interesante tratar de definir este concepto; soy consciente de que muchas de estas propuestas no pueden considerarse más que hipótesis de trabajo, válidas para circunscribir el marco de futuras investigaciones.

\section{CONCLUSIONES}

Las variables que tradicionalmente determinaban la realización de cualquier acto de consumo: necesidades, utilidad de los objetos, renta, condiciones sociales..., se han vuelto en la actualidad complejas y ambivalentes. Por eso una de las posibilidades más seguras que se ofrece a los educadores es tratar de comprender este proceso, partiendo del modo como se intercambian significados a través del consumo, viendo cómo los objetos se transmutan en mensajes y analizando los sujetos que interactúan socialmente en estas transacciones. El hombre posmoderno sin naturaleza, sin tiempo, sin historia ( F. Jameson 1985) ha quedado reducido a poco más que a consumidor de mensajes y la única forma de conocerlo es comprendiendo el modo como integra y se integra en la reproducción simbólica (J. Baudrillard 1978, S. Giner 1985 o P. Berger y H. Kellner 1985).

El consumo no puede entenderse únicamente como una satisfacción pasiva de necesidades, ni como un comportamiento automático conforme a los dictámenes del mercado, ni como una interpretación imaginaria de la realidad, ni como una interiorización cognitiva, ni como una conformación social. El consumo se expresa a través de un universo simulado y simbólico y expresa una determinada estratificación social. De ello, ha de ser consciente la escuela, mostrando al alumno cómo el consumo se origina hoy menos para satisfacer necesidades que para soportar significados socialmente creados.

Entendido así, el consumo precisa un análisis multidisciplinar e integrado, como el que ha originado esta monografía, capaz de valorar el modo como las distintas propuestas se complementan, teniendo en cuenta que, a través del consumo, no sólo se consumen productos y servicios, sino que también se asimilan conductas, modelos y estructuras sociales, construyén- 
dose, en fin, a los propios consumidores. Esta conclusión creo que tiene importancia pedagógica suficiente, para que sea considerada en cualquier programa de Educación para el Consumo.

En suma, toda acción de consumo es básicamente un acto de elección, que precisa una preparación y un aprendizaje, esto es una forma de responder a los mensajes que sustituyen a los objetos y simulan la realidad y por tanto, un modo de comunicación y de cultura. Todos los seres humanos están llenos de significados y tratan de vivir conforme a estos: el consumo simbólico es la fuente, o al menos uno de sus determinantes principales, de esta construcción simulada de la realidad, tal como he querido aclarar en mi artículo y tal como muestran otras aportaciones, especialmente las de P. Vidal y C. Hernández, de esta monografía.

\section{Notas}

1 «Una sociedad - dice en este sentido muy acertadamente D. L. Sayers en la que el consumo tiene que ser estimulado artificialmente a fin de mantener en funcionamiento la producción, es una sociedad fundada en la basura y el derroche, y una sociedad así es una casa construida sobre la arena." (Citado por V. Packard The Waste Makers, p. 8).

2 Conviene aclarar que existen otros múltiples modelos motivacionales, cuya naturaleza no es psicoanalítica (J. Arnau 1979); aunque de la «motivational research» se ha hecho uso y abuso en la investigación publicitaria (D. Victoroff 1980).

3 Tanto el conductismo como el psicoanálisis niegan la autonomía de la función cognitiva. Bien extrapolando la conducta animal a la humana en situaciones complejas como las que genera al consumo, o bien dando por supuesto que la motivación insconsciente proporciona el fundamento preciso para comprender las actitudes sociales. «Por supuesto - dice H. Tajfel- esto no significa que los factores motivacionales y emocionales sean poco importantes, pero también es cierto que la mayor ventaja adaptativa del hombres es su capacidad para modificar su conducta en función del modo como percibe y comprende una situación. (H. Tajfel 1984, pág. 158). 4 «Las actitudes - dice H. H. Kassarjian- son buenos predictores sólo cuando las medidas actitudinales y conductuales muestran un alto grado de correlación con la acción, el objetivo, el contexto y las variables de tiempo». (H. H. Kassarjian 1985, pág. 43).

5 Llamo consumo integrativo a la comprensión sociológica de este proceso porque evidencia el modo como el consumidor se identifica con cierros grupos, modos y estilos de vida o con toda la sociedad (P. Berger y $\mathrm{H}$. Hellner, 1985).

${ }_{6}$ «Pour la théorie économique - indican B. Brochand y J. Lendrevie en una línea muy parecida a la nuestral'achat et la consommation d'un produit s'expliquent par leur utilité, ç est-à-dire par leur valeur d'usage; pour la théorie psychologique par la nature et la force des motivations; pour la théorie sociale, l'explication est à rechercher dans la significacions sociale des objets» (B. Brochand y J. Lendrevie 1983, pág. 127).

7 Que fue la primera reflexión económica que integró al consumo como variable esencial de una economía en crisis. Una interpretación magistral de esta postura de J. M. Keynes es la que hace J. Robinson en los capítulos 25 y 26 de su obra La acumulación del capital.

8 «La societé de consommation - acota J. Baudrillard en este sentido- no se designe pas seulement par la profusion des biens et des services, mais par le fait, plus important, que TOUT EST SERVICE, que ce qui est donné à consommer ne se donne jamais comme produit pur et simple, mais bien comme service personnel, comme gratification.» (J. Baudrillard 1970, pág. 252).

\section{Resumen}

Publicidad y consumo simbólico es un articulo en el que se describe, en primer lugar, la complejidad que engloba boy cualquier conducta de consumo; para revisar, a continuación, los diversos modelos (económicos, psicológicos, sociológicos y comunicacionales) que han tratado de explicar este proceso, criticando sus limitaciones y proponiendo un nuevo enfoque, relacionado por el autor con el consumo simbólico.

Para fundamentar esta propuesta se revisan las diferentes variables que determinan el desarrollo de este proceso, matizando su funcionamiento y consecuencias sociales más inmediatas.

El artículo, cuyo objetivo principal es la descripción conceptual, termina con unas conclusiones en donde las propuestas planteadas se relacionan con el mundo de la educación y la cultura.

\section{Summary}

Advertising ans symbolic consumption is a paper in wich it is described, firstly, the complexity included, nowadays, in any consumption's behavior; thus, following with a review of different models (economical, psycho. 
logical, sociological, and communicating ones) which bave tried to explain this process, critizing theirs limitations and proposing a new approach, related to the symbolic consumption according to the author's opinion.

In order to ground this proposal, the different variables that determine this process development are reviewed, shading its functioning and most inmmediate social consequences.

The paper's main purpose is the conceptual description and it ends with some conclusions in wich the posed proposal appear related to the education and culture's world.

\section{Résumé}

Publicité et consommation symbolique est un article où l'on décrit, en premier lieu, la compléxité que toute conduite de consommation entraine de nos jours; pour réviser, à continuation, les différents modèles (économiques, psychologiques, sociologiques et communicateurs) qui ont essajé d'expliquer ce processus, en critiquant leurs limitations et en proposant une nouvelle approche, reliée avec la consommation symbolique, selon l'auteur.

Pour fonder cette proposition on revise les différentes variables qui déterminent le développement de ce processus, nuançant son fonctionnement et conséquences sociales plus inmédiates.

L'article, dont le but principal est la description conceptuelle, finit avec des conclusions où les propositions posées sont en relation avec le monde de l'éducation et la culture.

\section{Referencias}

AAker, D. A., y Morgan, S. M.: «Modelling store choice behavior», Journal of Marketing Research,8, 38-42. AjZen, I., y Fishbein, M.: Understanding Attitudes and Predicting Social Bebavior. Englowood Clifs: Prentice Hall, 1981.

Baudrillard, J.: Le système des objets, París: Gallimard, 1968.

Baudrillard, J: La societé de Consommation. París: Gallimard, 1970.

Braudrillard, J.: Pour une critique de l'economie politique du signe. París: Gallimard, 1972.

Brochand, B., y Lendrevie, J.: Le Publicitor. París: Dalloz, 1983.

Carrera, F.: «Aplicación publicitaria del modelo motivacional Expectativa-Valor», Publitecnia, 49-50, $27-49$.

Chatelat, B.: Les estiles de vie des Français, París: Stanke, 1980.

DiCHTER,: Handbook of Consumer Motivations: The psycbology of the world of Objets. Nueva York: McGrawHill, 1964.

Dogana, F.: Psicopatologia dei consumi quotidiani. Milán: Franco Angeli, 1980.

EhrenberG, A.S.C.: Repeat-buying. Theory and Application. Amsterdam y Nueva York: Nort Holland Publishing Co, 1972.

Engel, J. F., y Kollat, D.T., y Blackwell, R.D.: Consumer Behavior. New York: Holt Rinehart and Winston, 1968.

Fromm, E.: Psicoanálisis de la sociedad contemporánea. Buenos Aires: Paidós, 1960.

GalbRAITH, J.K.: La sociedad opulenta. Barcelona: Ariel, 1960.

GALBRAith, J. K.: El nuevo estado industrial. Barcelona: Ariel, 1967.

Galbrairh, J. K.: La era de las incertidumbres. Barcelona: Plaza y Janés, 1981.

Gonzalez MarTín, J. A.: Fundamentos para la teoría del mensaje publicitario. Madrid: Forja, 1982.

How ARD, J.A., y SETH, J. N.: The Theory of Buyer Bebavior. Nueva York: Wiley, 1969.

Green, H. A. J.: Consumer Theory, Middlesex: Penguin Books, 1971.

IвÁÑEZ, J.: «La publicidad en el capitalismo de consumo», Eroski, 1985, 2-15.

JACOBY, J.; HoYER, W.D., y SHEl,UGA, D.A.: «Viewer miscomprehension of televised communication: A brief report on finding», Adv. Consum Res, 8, 410-415.

KAPFFerer, J. N.: Les chemins de la persuasion. París: Bordas, 1978.

Kassarjian, H. N.: «Psicología del consumo», en Estudios de Consumo, 5, 37-60.

Katona, G.: The Powerful Consumer. Nueva York: McGraw Hill, 1960.

Katona, G.: Psychological Economics, Amsterdam: Elsevier, 1975.

LUTZ, R. J., y BETTMAN, J. R.: «Multiatribute models in marketing», Jacoby, 1977.

Marcus-StejIF, J.: Les Etudes de Motivation. París: Herman, 1961.

Martineau, P.: Un guide de la strategie Publicitaire. Motivation et publicité. París: Hommes et Techniques, 1969.

Miguel A. DE: Comprar en España. El punto de vista de los consumidores. Madrid: Instituto para la comunicación pública, 1983.

Reynaud, P.L.: Precis de Psychologie Economique. París: P.U.F. 1974.

TAJFEL, H.: Grupos bumanos y categorías sociales, Barcelona: Herder, 1984.

Veblen, T.: Teoria de la clase ociosa, México: F.C.E. 1944.

Veldhoven, J. M. van: Psichological Aspect of taxation. Ponencia presentada al V Congreso de Psicología Económica. Leuven (Bélgica), 1980.

WULF, D.: «Interpretation of Qualitative Material: A Matter of Intuition or a process to be Objetivitated?», Esomar, 1975. 